\title{
Statistical and systematic treatment issues in top quark mass combinations
}

\author{
Andreas Alexander Maier ${ }^{* \dagger}$ \\ Max-Planck-Institute for Physics \\ E-mail: andreas.alexander.maierecern.ch
}

\begin{abstract}
The past years have seen tremendous improvements in the precision of top quark mass measurements at hadron colliders. Since these measurements are mostly limited by systematic uncertainties, the achievable precision of a single measurement depends on developments in the experimental and theoretical understanding of top quark events. Complementing these efforts, a precision gain can also be obtained by a combination of measurements, exploiting their correlation. With ever increasing precision of top quark mass measurements from single experiments, this approach becomes more and more promising, because the absolute precision gain of refined techniques in both theory and experiment tends to saturate. This requires a precise matching of uncertainty categories and a detailed evaluation of the correlations of observables. This article reviews the status and points out the challenges faced in the ATLAS and CMS LHC top quark mass combination effort.
\end{abstract}

8th International Workshop on Top Quark Physics

14-18 September, 2015

Ischia, Italy

${ }^{*}$ Speaker.

${ }^{\dagger}$ On behalf of the ATLAS and CMS Collaborations 


\section{Introduction}

Alongside a thorough analysis of uncertainties to constrain their impact on a measurement, a sizeable precision gain can be obtained by a combination of measurements. This article discusses the challenges faced in the ATLAS [1] and CMS [2] LHC top quark mass combination effort.

Top quark mass combinations have usually been performed using the Best Linear Unbiased Estimator (BLUE) method [3], for example as described in references [4, 5]. The combined result is the linear combination of the inputs, minimising the total variance. For each measurement, the central values, the list of uncertainty components and the correlations of the estimators for each uncertainty component have to be provided. Consequently, a combination of measurements requires a precise matching of uncertainty categories and a detailed evaluation of the correlations of observables.

\section{Previous combinations}

Traditionally, combinations of top quark mass measurements are based on an a posteriori combination of published measurements. Various analysis differences like the choice of Monte Carlo (MC) simulation programs, uncertainty categorisation and analysis approaches complicate the determination of correlations. Therefore, the correlations of the measurements for all uncertainty categories are assigned based on physics arguments and varied within a reasonable range to assess the stability of the combination. This is in many cases the only possible way of combination, especially for older measurements, where the information on the specific analysis is often limited to the published material. In the last years, ATLAS, CMS, CDF and D0 have started to publish information relevant for a combination alongside the actual measurement. This comprises for example the publication of detailed components of the Jet Energy Scale (JES), $b$-tagging scale factor and Parton Distribution Function (PDF) uncertainties. The ATLAS and CMS collaborations move towards a harmonisation of uncertainty categorisation, for example of the JES components [6]. This facilitates the matching of uncertainty categories and leads to a more trustful correlation estimate.

An example among a series of recent combinations following this approach [7, 8, 9] is the world combination [10], including 11 measurements performed by the CDF and D0 experiments at Tevatron and the ATLAS and CMS experiments at LHC. The final result is $m_{\text {top }}=173.34 \pm$ 0.27 (stat) \pm 0.71 (syst) $\mathrm{GeV}=173.34 \pm 0.76 \mathrm{GeV}$, providing a $28 \%$ improvement with respect to the most precise single input measurement. Variations of the input uncertainties and correlations yield a remarkable stability of the results. The world combination in comparison to single measurement results and LHC combinations is shown in Figure 1.

\section{The hadronisation uncertainty at ATLAS and CMS}

An outstanding issue with respect to harmonisation of uncertainty categories between CMS and the other experiments is the treatment of the uncertainty related to parton shower and hadronisation effects, often denoted as hadronisation uncertainty. Traditionally, the uncertainty is estimated from the observed mass difference for samples produced with different parton shower generators, covering different parton shower concepts ( $p_{\mathrm{T}}$ or angular ordered), fragmentation functions, parameter tunes and the cluster or string hadronisation models. This introduces a certain amount of 


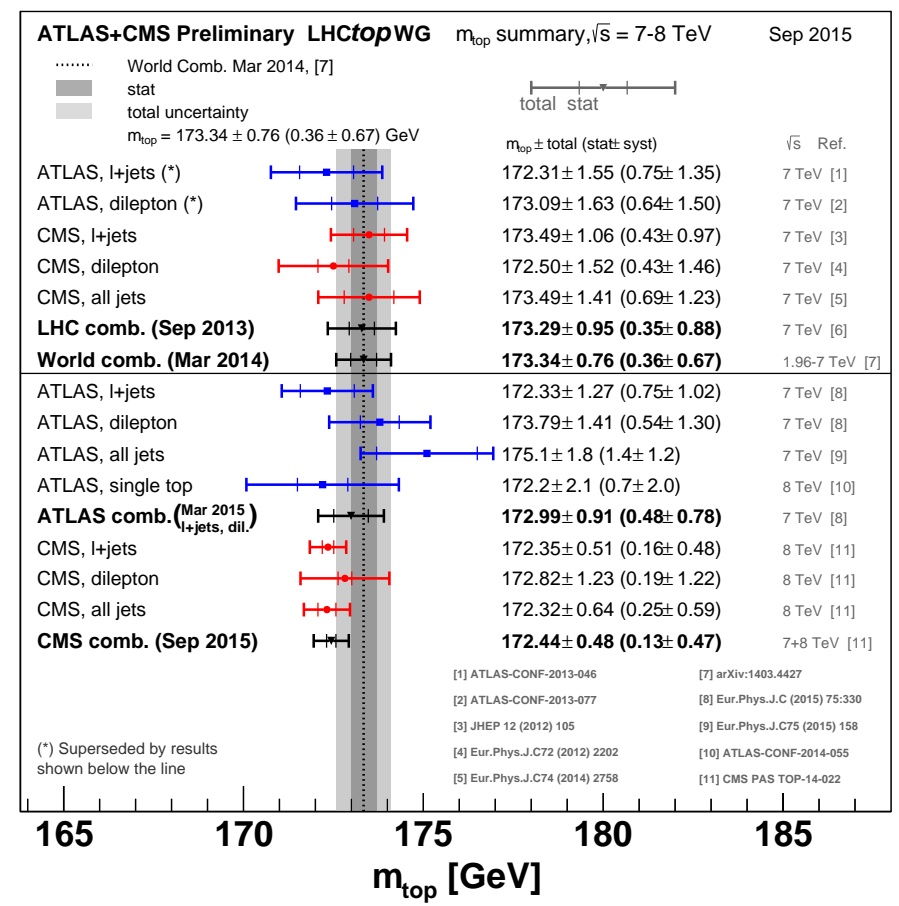

Figure 1: Recent LHC measurements and their combinations in comparison with the world combination of Tevatron and LHC experiments [11].

double-counting with the JES and the $b$-Jet Energy Scale (bJES) uncertainties, which also include effects of jet energy response differences from similar parton shower generator comparisons. The ATLAS and the Tevatron collaborations use the traditional approach, assuming, that the JES uncertainties do not cover the parton shower generator differences. This is motivated by the event topology differences of the $t \bar{t}$ events, used for the evaluation of the impact on the $m_{\text {top }}$ measurements, and the dijet and $Z$ boson events the JES uncertainty is determined from. A recent study from ATLAS shows that the amount of double-counting is indeed small, by eliminating the double-counting via a recalibration of jet responses [12]. The CMS collaboration avoids all double-counting and does not quote an explicit hadronisation uncertainty, assuming that the effects are fully covered by alternative uncertainties. These are a flavour dependent hadronisation uncertainty and an uncertainty covering variations of the $b$-fragmentation, $b$-hadron branching fractions and top quark $p_{\mathrm{T}}$ modelling [13]. This is supported by studies, indicating that differences between the Pythia and Herwig parton shower generators mainly stem from parton shower and tuning effects [14], rather than from different hadronisation models [15]. Not only is the impact of this uncertainty on the single analyses with $\mathscr{O}(0.5) \mathrm{GeV}$ relatively large, but its addition or removal also has a sizeable impact on the combination result. This underlines the high priority of unified uncertainty treatments for a combination of measurements.

\section{Determination of correlations at ATLAS and CMS}

Despite the significant reduction of the total uncertainty on $m_{\mathrm{top}}$, the approach of assigning correlations still comes with a severe drawback. Usually, large correlations are chosen, assuming 


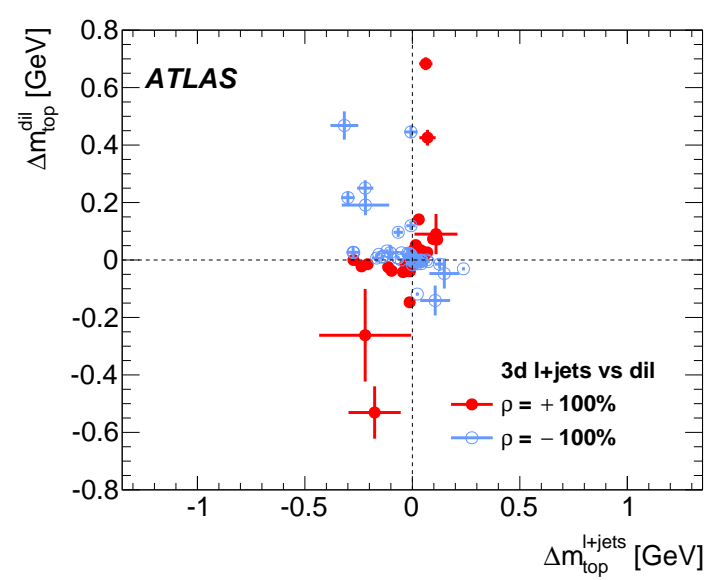

Figure 2: The systematic uncertainties of $m_{\text {top }}$ in the dilepton analysis versus those of the lepton+jets analysis [16]. Sources for which the two estimators are fully (anti-)correlated are shown in red (blue).

that this leads to a conservative estimate. This may not only be the wrong assumption in cases where a larger correlation is aggressive, but the corresponding final result is in general less precise. Additionally, negative correlations and the consequent mutual stabilisations, which may even lead to the complete insignificance of an uncertainty, are neglected.

At CMS, the correlation corresponding to a single systematic variation is assigned based on uncertainty ratios $\rho=\sigma_{1} / \sigma_{2}$, where the $\sigma_{1 / 2}$ denote the respective single measurement uncertainties [13]. This allows an unambiguous assignment of correlations $0<\rho<1$ and results in small correlations for differently sized systematic uncertainties. This approach is referred to as reduced correlation scenario. An assessment of this method is given in Reference [5]. The final result for the CMS combination of $\sqrt{s}=7$ and $8 \mathrm{TeV}$ measurements is $m_{\mathrm{top}}=172.44 \pm 0.13$ (stat) \pm 0.47 (syst) $\mathrm{GeV}=172.44 \pm 0.49 \mathrm{GeV}$, corresponding to a $4 \%$ gain in precision with respect to the most precise single measurement, which is the measurement in the lepton+jets channel.

At ATLAS, the correlations are determined based on systematic variations and the analysis of their effect on the measured $m_{\text {top }}$ value in the different measurements [16]. This is shown in Figure 2, where every point represents a systematic uncertainty variation together with the statistical precisions as uncertainty cross. The observed same or opposite sign $m_{\text {top }}$ shift in the measurements to be combined points at full correlation $(\rho=1)$ or full anti-correlation $(\rho=-1)$ of the analyses [16]. Correlations of composite uncertainties are evaluated by a summation of the covariance terms determined from the single component variation and a normalisation to the total uncertainty of the source. The final result for the ATLAS combination of $\sqrt{s}=7 \mathrm{TeV}$ measurements is $m_{\text {top }}=172.99 \pm 0.48$ (stat) \pm 0.78 (syst) $\mathrm{GeV}=172.99 \pm 0.91 \mathrm{GeV}$. This corresponds to a total correlation of $\rho=-7 \%$ and a $28 \%$ gain in precision with respect to the more precise single measurement, which is the measurement in the lepton+jets channel. With a thorough determination of correlations, the world combination's relative precision gain can thus be reached by the combination of only two measurements, which exhibit an even larger difference in the final untercertainty than the two most precise input measurements in the world combination. Assigning reduced correlations to the ATLAS measurements would result in a much larger total correlation of $\rho=33 \%$. This demonstrates that the determination of correlations yields a potentially larger gain in precision than an assignment, due to the exploitation of anti-correlations. 


\section{Conclusion}

A detailed understanding of correlations of observables based on a well motivated uncertainty mapping across experiments is the key to successful combinations. The unification of uncertainty categories in ATLAS and CMS is progressing. A way of determining the correlation of measurements from systematic variations has been developed, leading to a more reliable correlation estimate than the method of correlation assignment used in previous combinations. The measurements at $\sqrt{s}=8 \mathrm{TeV}$ are waiting to be combined and a sizeable gain in precision is expected [17].

\section{References}

[1] ATLAS Collaboration, The ATLAS Experiment at the CERN LHC, JINST. 3 (2008) S08003.

[2] CMS Collaboration, The CMS experiment at the CERN LHC, JINST 3 (2008) S08004.

[3] L. Lyons et al., How to combine correlated estimates of a single physical quantity, Nucl. Instr. Meth. A270 (1988) 110.

[4] R. Nisius, A ROOT class to combine a number of correlated estimates of one or more observables using the Best Linear Unbiased Estimate method, http: / / blue. hepforge.org/Bluemanual .pdf.

[5] R. Nisius, On the combination of correlated estimates of a physics observable, EPJC 74 (2014) 3004.

[6] ATLAS and CMS Collaborations, Jet energy scale uncertainty correlations between ATLAS and CMS, ATL-PHYS-PUB-2014-020, 2014, https://cds . cern. ch/record/1956734.

[7] ATLAS and CMS Collaborations, Combination of ATLAS and CMS results on the mass of the top quark using up to $4.9 \mathrm{fb}^{-1}$ of data, ATLAS-CONF-2013-102, 2013, http: / / cds. cern. ch/record/1601811.

[8] Tevatron Electroweak Working Group, CDF and D0 Collaborations, Combination of CDF and D0 results on the mass of the top quark using up to $9.7 \mathrm{fb}^{-1}$ at the Tevatron, arXiv:1407.2682.

[9] CMS Collaboration, Combination of the CMS top-quark mass measurements from Run 1 of the LHC, CMS-PAS-TOP-14-015, 2014, https://cds . cern.ch/record/1951019.

[10] ATLAS, CDF, CMS and D0 Collaborations, First combination of Tevatron and LHC measurements of the top-quark mass, arXiv: 1403.4427.

[11] ATLAS Collaboration, Summary plots from the ATLAS Top physics group, November, 2015, https://atlas.web. cern. ch/Atlas/GROUPS / P HYS ICS / CombinedSummaryPlots / TOP /.

[12] ATLAS Collaboration, Impact of fragmentation modelling on the top quark mass measurement using the ATLAS detector, ATL-PHYS-PUB-2015-042, 2015, https : / / cds.cern.ch/record/ 2054420.

[13] CMS Collaboration, Measurement of the top quark mass using proton-proton data at $\sqrt{s}=7$ and $8 \mathrm{TeV}$, arXiv:1509.04044.

[14] M. Seidel, Precise measurement of the top-quark mass at the CMS experiment using the ideogram method, $\mathrm{PhD}$ thesis, DESY-THESIS-2015-034, 2015, https : / / bib-pubdb1.desy . de/record/222219.

[15] B. Stieger, Towards a new top mass world combination, Talk at LHCTopWG meeting, May 2015, https://indico.cern.ch/event/375429.

[16] ATLAS Collaboration, Measurement of the top quark mass in the $t \bar{t} \rightarrow$ lepton + jets and $t \bar{t} \rightarrow$ dilepton channels using $\sqrt{s}=7 \mathrm{TeV}$ ATLAS data, EPJC 75 (2015) 330

[17] G. Cortiana, Top-quark mass measurements: review and perspectives, arXiv:1510 . 04483. 